\title{
Costos y rentabilidad de la apicultura a pequeña escala en comunidades mayas del Litoral Centro de Yucatán, México
}

\author{
Cost and profitability of small scale beekeeping in center coast \\ mayan communities of Yucatan, Mexico
}

Lucely C. Contreras Uc*, Miguel A. Magaña Magaña*®

\begin{abstract}
Contreras Uc, L. C., \& Magaña Magaña, M. A. (2017). Costos y rentabilidad de la apicultura a pequeña escala en comunidades mayas del Litoral Centro de Yucatán, México. Investigación y Ciencia de la Universidad Autónoma de Aguascalientes, 25(71), 52-58.
\end{abstract}

\section{RESUMEN}

El objetivo del presente estudio fue determinar y analizar la magnitud y estructura de los componentes relacionados con la rentabilidad del proceso de producción apícola. La información primaria se obtuvo a través de una encuesta por muestreo estadístico estratificado, el marco lista se integró con los productores de nueve localidades del municipio de Motul, considerado representativo de la región Litoral Centro de Yucatán, México. Se encontró que la estructura porcentual de los costos de producción de la miel se compone mayoritariamente por el costo variable, cuyos principales rubros fueron el valor del azúcar y el desembolso por mano de obra. Por su parte, el costo fijo lo constituyó principalmente la depreciación en infraestructura. La ganancia fue positiva en los estratos considerados; el rendimiento y el precio de la miel, determinantes del ingreso, fueron los principales elementos de la variación en la rentabilidad que se obtiene en el proceso de producción apícola.

\section{ABSTRACI}

The objective of this study was to determine and analyze the magnitude and structure of the components

Palabras clave: costos de producción; ingresos; rentabilidad; apicultura; precio de venta.

Keywords: production costs; income; profitability; beekeeping; sale price.

\section{Recibido: 22 de julio de 2016, aceptado: 20 de octubre de 2016}

* División de Estudios de Posgrado e Investigación, Instituto Tecnológico de Conkal. Avenida Tecnológico s/n, C. P. 97345, Conkal, Yucatán, México. Correo electrónico: lucelycontreras@gmail.com; drmmagana@

gmail.com

$凶$

Autor para correspondencia related to the profitability of the beekeeping production process. The primary information was obtained through a survey by statistical stratified sampling, the framework list was integrated with the producers' name that belongs to nine villages of the municipality in Motul, regarded as representative of the Coast Center in Yucatan State, Mexico. It was found that the percentage structure of honey production costs is composed mainly by the variable cost, whose main items were the value of sugar and the disbursement by labor. On the other hand, fixed cost was mainly the depreciation in infrastructure. The gain was positive in the considered strata; the performance and price of honey, determinants of income were the main components of the change in the profitability obtained in the process of beekeeping.

\section{INTRODUCCIÓN}

La cría de abeja ha estado presente desde el México prehispánico, en el inicio con la Melipona beecheii y a mediados del siglo XX se introdujo la Apis mellifera ligustica (Munguía, 1999), con este cambio la actividad adquirió una mayor importancia técnica y económica, ya que los productores adoptaron nuevas formas de manejo, mayor nivel de ingreso monetario y respondieron a la demanda del mercado internacional (Rosales González, \& Rubio Herrera, 2010).

En el estado de Yucatán, México, la apicultura se práctica generalmente en unidades de producción a pequeña escala (Magaña Magaña, Aguilar Arrieta, Lara y Lara, \& Sanginés García, 2007), la cual proporciona beneficios económicos a los productores (García Gómez, \& Meza Ramos, 2012). La rentabilidad que genera es un elemento importante en la estrategia económica y de vida de las familias del me- 
dio rural (Pat, Cortés, \& Calderón, 2014). Al respecto, Magaña Magaña, Moguel Ordóñez, Sanginés García y Leyva Morales (2012) mencionan que el ingreso por venta de miel, los salarios y el valor de la compra de insumos, equipos y materiales, son los principales rubros del efecto multiplicador del ingreso que genera esta actividad sobre la región. Por su parte, Martínez (2010) expone que en Yucatán la explotación apícola ha generado una industria familiar, lo que ha permitido el arraigo de los productores en sus lugares de origen, debido a que se evita la migración por falta de trabajo.

Esta actividad primaria enfrenta problemas socioeconómicos y técnicos, los cuales han tenido considerables consecuencias en la estructura de los costos y, por ende, en los beneficios económicos (Güemes Ricalde, \& Villanueva Gutiérrez, 2002). Entre algunos problemas específicos pueden mencionarse la falta de adopción de tecnologías actuales acordes a las exigencias de inocuidad del mercado; las formas de comercialización, principalmente hacia el exterior, donde solo participan exportadores con un esquema de intermediarismo (Ulloa-Castañeda et al., 2014). Aunado a lo expuesto se encuentra el cambio climático que a su vez ha provocado modificaciones en la temperatura, la precipitación pluvial y la modificación de estaciones, lo que afecta negativamente la producción (Ulloa-Castañeda, Meza-Ramos, Arnaldo-Velázquez, Aguirre-Ortega, \& Martínez-Virgen, 2014). Al respecto, Medina-Cuéllar, Portillo-Vázquez, García Álvarez-Coque, Terrazas-González y Alba-Nevárez (2014) mencionan que las fluctuaciones climáticas son en gran parte responsables de la variabilidad interanual del rendimiento de miel, dada su influencia en la fenología tanto de la fuente de néctar como de las abejas. Al variar la producción apícola, la rentabilidad sufre el mismo cambio, esto aun existiendo una tendencia alcista en los precios (Salas, 1988).

El propósito del presente estudio fue estimar y determinar el estado de resultados de la actividad apícola a pequeña escala, con el fin de analizar los componentes asociados al nivel de rentabilidad y contribuir a la escasa literatura existente.

$$
\text { MATERIALES Y MÉTODOS }
$$

La información base del presente trabajo se obtuvo mediante una encuesta por muestreo estadístico a productores apícolas del municipio de Motul, considerado como caso de estudio representativo de la región maya del Litoral Centro de Yucatán, el cual comprendió parte de la anterior zona henequenera. En la encuesta se utilizó una cédula de entrevista con diversos apartados como la tenencia de colmenas, prácticas de manejo, infraestructura e información económica. La muestra preliminar se integró al azar con $30 \%$ del marco lista integrado por productores de la cabecera del municipio de interés y de las localidades de Kaxatah, Kiní, Ucí, Santa Teresa, Dzununcán, Santa Cruz Pachón, Kopté y San Pedro Chacabal.

En la estimación de la muestra final de apicultores se empleó la técnica de muestreo estratificado (Scheaffer, Mendenhall, \& Ott, 2007) y se consideraron tres categorías: productores que explotaban hasta 25 colmenas, de 26 a 50 y de 51 a 100 colonias. La variable asociada al muestreo, que permitió la estimación de la varianza en la muestra e indica la capacidad de producción fue el número de colmenas y el límite de error de estimación fue $5 \%$ del valor de la citada tenencia promedio. La muestra final fue de 55 productores, inferior al de la muestra preliminar. A la primer categoría le correspondió 58 \% de la muestra, $28 \%$ a la segunda y $14 \%$ a la tercera.

La información fue registrada y procesada en una hoja de cálculo del programa Excel. Las principales variables económicas (costos variables, fijos, ingresos, rentabilidad y coeficiente de rentabilidad) se estimaron siguiendo los procedimientos propuestos por Baca Urbina (2010). Para determinar el grado de asociación entre rentabilidad y sus componentes se recurrió a un modelo de regresión lineal múltiple, cuyas variables explicativas fueron los elementos del costo e ingreso. Los parámetros estimados de la misma son los siguientes $(R \wedge 2=0.96)$ :

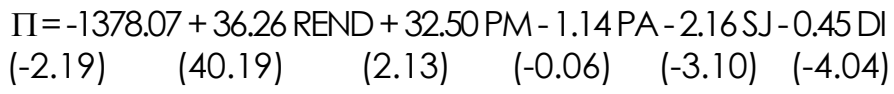

Donde:

$\Pi=\quad$ Rentabilidad por colmena

REND $=$ Rendimiento de miel por colmena

$\mathrm{PM}=$ Precio de la miel por $\mathrm{kg}$

$\mathrm{PA}=\quad$ Precio del azúcar por $\mathrm{kg}$

$\mathrm{SJ}=\quad$ Salario promedio por jornada

$\mathrm{DI}=\quad$ Valor promedio por colmena de la depreciación de infraestructura

El valor entre paréntesis representa el estadístico † 
Tabla 1

Inversión realizada por apiario

\begin{tabular}{lcccc}
\hline & \multicolumn{2}{c}{ Estratos de productores por número de } & \multicolumn{2}{c}{ colmenas } \\
\cline { 2 - 4 } Concepto & $\begin{array}{c}\text { 1 a } 25 \\
(\$)\end{array}$ & $\begin{array}{c}26 \text { a } 50 \\
(\$)\end{array}$ & $\begin{array}{c}50 \text { a } 100 \\
(\$)\end{array}$ & $\begin{array}{c}\text { Promedio } \\
\text { general } \\
(\$)\end{array}$ \\
\hline Equipo de protección y trabajo & $4,673.1$ & $9,590.8$ & $10,797.8$ & $7,082.5$ \\
\hline Infraestructura en campo & $7,364.1$ & $7,429.3$ & $9,462.1$ & $7,962.2$ \\
\hline Total de inversión & $12,037.2$ & $17,020.1$ & $20,259.9$ & $15,044.7$ \\
\hline
\end{tabular}

\section{RESULTADOS}

\section{Inversiones en infraestructura y equipo}

Las unidades de producción apícola del Litoral Centro de Yucatán están integradas por las colmenas tradicionales, con una base o soporte que sostiene los cuerpos, el piso y piquera de madera; cámara de cría con 10 cuadros en su interior; alzas y tapa. La inversión por apiario se presenta en la tabla 1.

La inversión en infraestructura en campo, que comprende las piletas o bases sobre las cuales se colocan soportes que sostienen las rieles, tubos o maderos con función de tarima, los cubos o cajas para cámara de cría y alzas que se acomodan sobre la citada tarima; tapas, pisos y cuadros, cuyo valor total, a medida que la capacidad productiva del apicultor incrementa, va perdiendo importancia con respecto a la inversión que se realiza en equipo de trabajo y seguridad.

El hecho citado anteriormente se observa a partir del segundo estrato, debido, por ejemplo, a que la calidad del material de los equipos adquiridos, como el de extracción, se relaciona con el nivel tecnológico que se emplea en la unidad de producción apícola, así como con las exigencias de inocuidad que dictan los mercados internacionales. Se observa que el inventario o posesión de equipos complementarios, como extractor, banco desoperculador, embudo, cuchillos, entre otros, presentan un aumento progresivo con el tamaño del apiario; situación similar exhibe la calidad del material de los mismos.

\section{Estructura del costo de producción}

La estructura porcentual de los costos de producción se compone mayoritariamente del costo variable, cuya contribución al total asciende a $76.3 \%$ para el primer estrato, $78.3 \%$ para el segundo y $81.2 \%$ para el tercero; mientras que el costo fijo participa con $23.7 \%$, $21.7 \%$ y $18.8 \%$ por categoría, en el orden citado ante- riormente; estos porcentajes evidencian una relación inversa con la capacidad de producción o de tenencia de colmenas por productor.

Con respecto a la participación relativa de las erogaciones en los costos variables del sistema de producción de interés, puede mencionarse que en ellas contribuyen en primera instancia el valor de compra del azúcar (tabla 2), insumo utilizado en esta

Tabla 2

Estructura porcentual del costo de producción por apiario

\begin{tabular}{|c|c|c|c|c|}
\hline \multirow{2}{*}{ Concepto } & \multicolumn{3}{|c|}{$\begin{array}{l}\text { Estratos de productores por } \\
\text { número de colmenas }\end{array}$} & \multirow{2}{*}{$\begin{array}{c}\text { Promedio } \\
\text { general } \\
\text { (\%) }\end{array}$} \\
\hline & $\begin{array}{l}1 \underset{(\%)}{a} 25 \\
(\%)\end{array}$ & $\begin{array}{l}26 \text { a } 50 \\
(\%)\end{array}$ & $\begin{array}{c}50 \text { a } 100 \\
(\%)\end{array}$ & \\
\hline \multicolumn{5}{|l|}{$\begin{array}{l}\text { COSTOS } \\
\text { VARIABLES: }\end{array}$} \\
\hline Azúcar & 36.8 & 39.3 & 42.4 & 38.7 \\
\hline Medicamentos & 14.7 & 14.8 & 15.8 & 15.0 \\
\hline Cera & 4.7 & 4.9 & 4.6 & 4.7 \\
\hline Mano de obra & 24.5 & 22.8 & 21.5 & 23.3 \\
\hline Flete & 10.8 & 9.5 & 1.9 & 8.7 \\
\hline Mant. vehículo & 7.5 & 8.6 & 12.4 & 8.8 \\
\hline Renta extractor & 0.5 & 0.0 & 0.0 & 0.2 \\
\hline Compra reinas & 0.5 & 0.2 & 1.3 & 0.5 \\
\hline TOTAL: & 76.3 & 78.3 & 81.2 & 77.9 \\
\hline \multicolumn{5}{|l|}{ COSTOS FIJOS: } \\
\hline $\begin{array}{l}\text { Deprecia. } \\
\text { equipo }\end{array}$ & 22.7 & 28.8 & 37.9 & 27.2 \\
\hline Deprecia. infraest & 65.5 & 51.5 & 38.5 & 56.4 \\
\hline $\begin{array}{l}\text { Deprecia. } \\
\text { vehículo }\end{array}$ & 11.7 & 19.6 & 23.6 & 16.4 \\
\hline TOTAL: & 23.7 & 21.7 & 18.8 & 22.1 \\
\hline $\begin{array}{l}\text { COSTO TOTAL DE } \\
\text { PRODUCCIÓN }\end{array}$ & 100.0 & 100.0 & 100.0 & 100.0 \\
\hline
\end{tabular}

Nota: Elaboración propia. 


\section{InVESTIGACIÓn Y CIEnCIA DE LA UחIVERSIDAD AUTÓnOMA DE RGUASCALIEחTES}

región y en otras para proveer a las colonias un alimento artificial; el cual tiene una tendencia progresiva a medida que aumenta el número de colmenas. Este hecho se asocia generalmente a las ineficiencias del manejo alimenticio, ya que se observó que cuando la capacidad instalada aumenta y se carece de otros insumos para la nutrición artificial, se recurre generalmente al azúcar.

El segundo rubro de costos que tiene una participación porcentual de casi una cuarta parte de la estructura del costo variable corresponde al desembolso por mano de obra; el cual presenta una disminución cuando el número de colmenas incrementa, hecho que evidencia el uso eficiente del recurso humano. Por su parte, la erogación realizada en medicamentos, principalmente para el control del ácaro Varroa destructor, es el tercer rubro en la estructura del costo variable y su valor porcentual presenta una relación directa con la tenencia de colmenas. En su conjunto, los tres principales rubros de costos variables mencionados anteriormente constituyen $60 \%$ del total de la estructura porcentual del costo total de producción.

Con referencia al segundo componente de los costos totales de producción, los costos fijos, se integran en mayor proporción por el valor de la depreciación de infraestructura en campo, seguido por la de los equipos de trabajo y protección. La depreciación de los materiales y la de vehículos presentaron una relación directa con la tenencia de colmenas; ocurre lo opuesto con la depreciación de infraestructura.

\section{Ingresos y rentabilidad}

El estado de resultados del proceso productivo y su correspondiente análisis es una herramienta que facilita la objetiva y oportuna toma de decisiones con respecto a las actividades agropecuarias, donde la producción está supeditada a factores de diversa índole. El ingreso de los apicultores de las comunidades mayas está determinado principalmente por el valor obtenido de la venta de miel. En menor proporción, en esta variable se consideró el valor obtenido por la venta de otros productos de la colmena. Los ingresos por apiario ascienden a 20,096.9 pesos para el primer estrato, 38,187.1 pesos y $\$ 40,020.6$ para el segundo y tercer estrato (tabla 3); la participación relativa de la miel en dicho agregado fue de $99.5 \%$.

Por su parte, se encontró que los precios de venta de miel por $\mathrm{kg}$ se diferencian según el tipo de floración predominante de cuyo néctar es extraído el dulce; así, para la floración del tajonal (Vigueira dentata) y el tzizilché (Gymnopodium floribundum) fue de 40.00 pesos, para el jabín (Piscidia piscipula) de 38.00 pesos, para las enredaderas de 36.00 pesos y para el tzalam (Lysiloma latisiliquum) tal valor fue de 34.00 pesos. El precio promedio ponderado se estimó en 38.40 pesos. Es importante mencionar que las estimaciones están regidas por los intermediarios locales, quienes supeditan la compra e imponen las condiciones de la misma.

En cuanto a la ganancia obtenida del proceso de producción apícola, se obtuvo un saldo positivo; es decir, los costos totales de producción fueron menores al ingreso obtenido por la venta de miel y cera. El superávit promedio en los tres estratos fue de 16,723.7 pesos; lo anterior se refleja en el coeficiente de rentabilidad, el cual tuvo un promedio de $28.6 \%$. Por su parte, la rentabilidad y su coeficiente siguen un comportamiento directo con la tenencia de colmenas (tabla 3), lo que evidencia un uso eficiente de los recursos al aumentar la capacidad de producción.

Tabla 3

Costo de producción y rentabilidad de la actividad apícola por apiario

\begin{tabular}{lcccc}
\hline \multirow{2}{*}{\begin{tabular}{l} 
Concepto \\
\cline { 2 - 5 }
\end{tabular}} & \multicolumn{2}{c}{ Estratos de productores por número de colmenas } & Promedio \\
\cline { 2 - 5 } & 15.1 & 26 a 50 & 50 a 100 & 20.7 \\
\hline Colmenas/apiario & $8,896.4$ & $14,663.6$ & $14,113.4$ & $11,247.5$ \\
\hline Costo tot. de prod (\$) & $20,096.9$ & $38,187.1$ & $40,020.6$ & $27,971.2$ \\
\hline Total de ingresos (\$) & $11,200.5$ & $23,523.5$ & $25,907.2$ & $16,723.7$ \\
\hline Rentabilidad (\$) & 26.3 & 30.6 & 33.8 & 28.6 \\
\hline Coef. rentabilidad (\%) & & & &
\end{tabular}


IIVESTIGACIÓn Y CIERCIA DE LA UNIVERSIDAD AUTÓNOMA DE RGUASCALIERTES
Lo anterior contrasta con la tendencia que siguen los costos de producción con respecto al tamaño de la explotación.

Con relación a la influencia que ejercen las variables rendimiento, precio de la miel, costo del azúcar, salario por jornada y valor de la depreciación de infraestructura sobre la rentabilidad, se encontró con base en los parámetros estimados de la regresión lineal múltiple, que la variable rendimiento explica una variación positiva de 36.26 pesos en la rentabilidad al cambiar en una unidad ( $\mathrm{kg} / \mathrm{colmena}$ ). Esta variable resultó la de mayor influencia sobre la rentabilidad.

La segunda variable que ejerce una influencia considerable sobre la rentabilidad es el precio de la miel, ya que al variar la misma en una unidad (\$) la rentabilidad varía positivamente en 32.5 pesos. Por su parte, el precio del azúcar, el salario por jornada y el valor de la depreciación ejercen una limitada e inversa influencia sobre la rentabilidad. Así, al variar la primera variable en una unidad (\$) la disminución de la rentabilidad es de 1.14 pesos; mientras que la variación en una unidad (\$) en el valor del salario por jornada ocasiona un decremento de 3.1 pesos en la variable dependiente. Para la tercera variable, el valor de la depreciación de infraestructura, se determinó que una variación en la misma de una unidad (\$) disminuye en 4.04 pesos la rentabilidad.

La evidencia proporcionada por el modelo de regresión lineal múltiple sugiere que las variables rendimiento por colmena y precio de la miel, principales factores determinantes del ingreso, resultaron ser las variables que explican los mayores cambios en la rentabilidad de la actividad apícola.

\section{DISCUSIÓN}

Dolores-Mijangos (2014) reportó que en la región húmeda del Istmo de Tehuantepec, Oaxaca, México, la inversión en infraestructura productiva y equipos por colmena fue en promedio 1,248.9 pesos para productores con 23 y 105 colmenas; lo cual fue similar a lo que se estimó en las comunidades mayas del Litoral Centro de Yucatán, donde el promedio general de inversión por colmena fue de 1,201.3 pesos.

Magaña Magaña et al. (2007) encontraron que el nivel del costo de producción de $1 \mathrm{~kg}$ de miel evidencia una relación inversa con el número de colmenas en posesión, lo cual fue similar a lo hallado en el presente estudio, ya que para el primer estrato de productores el costo total de producción por kilogramo de miel fue de 19.7 pesos. Para el segundo fue de 16.6 pesos, mientras que para el tercero, de 14.4. La citada relación inversa obedece principalmente al uso más eficiente de los insumos y factores de la producción, así como de los activos fijos.

Con relación a la estructura del costo de producción, Ichazo, Kaegi, Salas y Sanabria (1991) en Honduras encontraron que el costo fijo representa $40 \%$ de tal estructura, mientras que en Oaxaca, Dolores-Mijangos (2014), encontró que los costos variables tienen una participación de $65.4 \%$ y $73.0 \%$ en la estructura de los costos para productores con 23 y 105 colmenas en promedio, respectivamente. Lo encontrado por dichos autores fue similar a lo estimado en las comunidades del Litoral Centro de Yucatán. Por su parte, Ulloa et al. (2014) reportaron para los productores de Nayarit que los costos fijos representaron en promedio $67 \%$ y $80 \%$ de la estructura de los costos de producción, por lo que el costo variable fue menor, lo cual difiere con lo encontrado en el estudio; esta diferencia obedece a la limitada tenencia de equipos, instalaciones y vehículos por parte de los apicultores del Litoral Centro de Yucatán, cuya disponibilidad de activos fijos se asocia al bajo nivel tecnológico.

En Argentina, Ulmer, Travadelo, Caporgno y Castignani (2011) reportaron que el valor de venta de miel representó alrededor de $82 \%$ en el ingreso total de los productores; la venta de otros productos de la colmena tales como núcleos, cera, celdas reales y reinas, complementan dicho rubro. Con esto los productores intentaron mejorar los indicadores económicos y financieros de la empresa. Los resultados anteriores son coincidentes en parte con lo encontrado en las comunidades mayas del Litoral Centro de Yucatán (figura 1), donde lo similar fue que la miel representó el producto que contribuyó en mayor medida al ingreso y la diferencia está en la escasa diversificación, ya que por lo general se obtiene un segundo producto de la colmena, la cera.

En Guatemala, Molina Reyes (2010) halló que los precios de venta, así como las condiciones de mercado y otros factores económicos son fijados por los intermediarios, por lo cual los apicultores no reciben valores significativos que aumenten su rentabilidad. Lo mencionado por dicho autor concuerda con lo encontrado en el estudio, circunstancia que repercute sobre el nivel de los ingresos. 


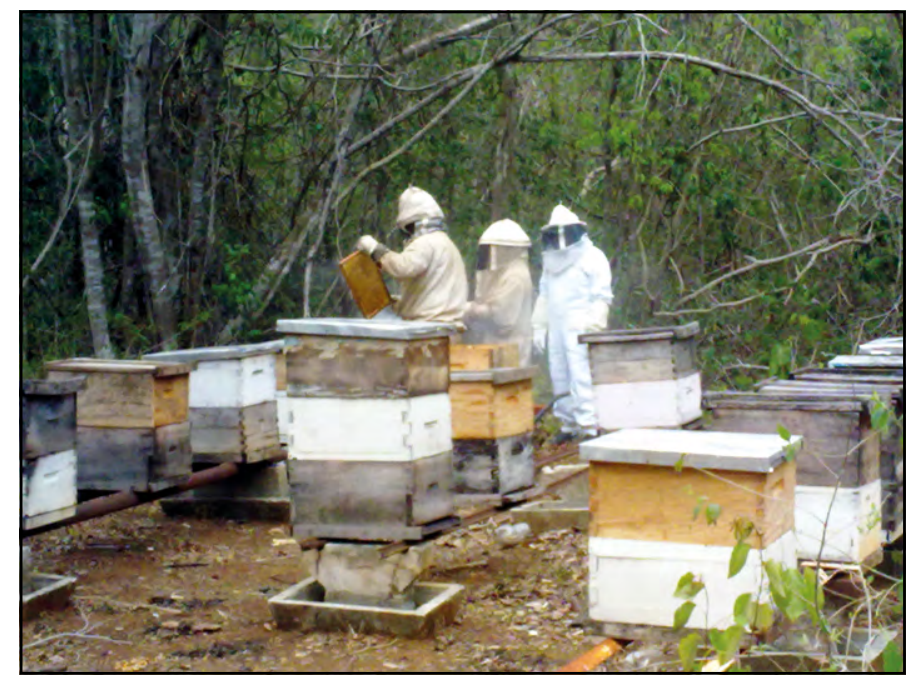

Figura 1. Apicultores en revisión de colmenas previa a la cosecha en Yucatán, México.

Fotografía proporcionada por los autores.

Magaña Magaña y Leyva Morales (2011) encontraron a través de un modelo de regresión que el precio de venta y la productividad fueron las principales variables explicativas de la rentabilidad apícola. Asimismo, concluyeron que la causa principal de la probable variación en el nivel de rentabilidad de las explotaciones apícolas en el territorio nacional lo constituye la caída del ingreso por la venta de miel. Lo anterior fue similar a lo hallado en el Litoral Centro de Yucatán, ya que los precios pagados al api-

\section{InVESTIGACIÓn Y CIERCIR DE LA UกIVERSIDAD AUTÓOOOMA DE RGUASCALIERTES}

cultor, impuesto por los intermediarios, así como la productividad por colmena, son las dos variables que constituyen el nivel del ingreso, que dado el costo de producción, determinan el nivel de rentabilidad correspondiente.

\section{CONCLUSIONES}

La rentabilidad que obtienen los apicultores de las comunidades mayas de la región Litoral Centro de Yucatán no está garantizado que sea positiva debido a que el precio de los insumos aumentan de manera constante, mientras que los rendimientos por colmena varían con relación al manejo técnico y a los factores climáticos adversos, además de que el precio de venta está sujeto a las condiciones que imponen los intermediarios locales. Esta situación ocasiona que los ingresos fluctúen regularmente. A pesar de lo anterior, se encontró que los ingresos del apicultor, determinados principalmente por los precios de venta de la miel, fueron superiores a los costos de producción, por lo que la actividad apícola en la región de interés presentó un superávit con márgenes elevados, mismo que es aprovechado por los productores para invertir en infraestructura básica, como equipo de extracción, que ante las exigencias imperantes de los países importadores se ven en la necesidad de modernizar tanto sus equipos como sus prácticas de manejo de la colonia, esto para cumplir con las normas de inocuidad de la miel.

\section{REFERENCIAS}

- Baca Urbina, G. (2010). Evaluación de proyectos. (6 ${ }^{a}$ ed.). México: McGraw-Hill.

- Dolores-Mijangos, G. (2014). La apicultura como una alternativa complementaria para incrementar los ingresos en las unidades de producción rural en la región húmeda del Istmo de Tehuantepec, Oaxaca. Tesis. Colegio de Postgraduados.

- García Gómez, L. E. \& Meza Ramos, E. (2012). Oportunidades y obstáculos para el desarrollo de la apicultura en Nayarit. Tepic, Nayarit, México. Recuperado de http://www.eumed.net

- Güemes Ricalde, F. J., \& Villanueva Gutiérrez, R. (2002). Características de la apicultura en el estado de Quintana Roo y del mercado de sus productos. Chetumal, Quintana Roo, México: Gobierno del estado de Quintana Roo-Sisierra-UQrooEcosur.

- Ichazo-S., L. H., Kaegi, O., Salas, R., \& Sanabria, O. (1991). Estudio de costos de producción para miel y polen en apicultura migratoria de la Escuela Agrícola Panamericana. Tesis. Honduras: Escuela Agrícola Panamericana. Recuperado de http://hd1.handle.net/1 1036/4291

- Magaña Magaña, M. A., \& Leyva Morales, C. E. (2011). Costos y rentabilidad del proceso de producción apícola en México. Contaduría y Administración, 235, 99-119.

- Magaña Magaña, M. A., Aguilar Arieta, A., Lara Lara, P., \& Sanginés García, R. (2007). Caracterización socioeconómica de la actividad apícola en el estado de Yucatán, México. Agronomía, 15(2), 17-24.

- Magaña Magaña, M. A., Moguel Ordóñez, Y. B., Sanginés García, J. R., \& Leyva Morales, C. E. (2012). Estructura e importancia de la cadena productiva y comercial de la miel en México. Revista Mexicana de Ciencias Pecuarias, 3(1), 49-64.

- Martínez, S. (2010). Plan rector sistema producto apícola de Oaxaca (p.13). México: Universidad Autónoma Chapingo. 
- Medina-Cuéllar, S. E., Portillo-Vázquez, M., García ÁlvarezCoque, J. M., Terrazas-González, G. H., \& Alba-Nevárez, L. L. (2014). Influencia del ambiente sobre la productividad de la segunda cosecha de miel de abeja en Aguascalientes de 1998 a 2010. Revista Chapingo, serie Ciencias Forestales y del Ambiente, 20(2), 159-165.

- Molina Reyes, A. O. (2010). Tipificación de los sistemas de producción apícola de siete municipios del departamento de Huehuetenango. Tesis. Guatemala: Universidad de San Carlos de Guatemala.

- Munguía, G. M. (1999). La experiencia de organización de los pequeños productores de miel de América Latina a partir del trabajo conjunto de EDUCE y Kabitah en Campeche, PAUAL. En Memorias del Primer Foro de Proyectos Integrales: Sistema Producto Miel. Mérida, Yucatán, México: Conacyt-SisierraUADY

- Pat, F. L. A., Cortez, C. O. M., \& Calderón, G. G. (2014). Rentabilidad y alternativas de comercialización de los pequeños productores de miel en Campeche, México. XIX Congreso Internacional de Contaduría, Administración e Informática.
- Rosales González, M., \& Rubio Herrera, A. (2010). Apicultura y organizaciones de apicultores entre los mayas de Yucatán. Estudios de cultura maya, 35, 163-186.

- Salas, W. (1988). Economía agrícola aplicada a la apicultura: Una propuesta metodológica para el análisis de la actividad. Agronomía costarricense, 12(2), 231-236.

- Scheaffer, R. L., Mendenhall, W., \& Ott, R. L. (2007). Elementos de muestreo. (6 $6^{a}$ ed.). Madrid, España: International Thomson Editores Spain Paraninfo.

- Ulloa-Castañeda, R. R., Meza-Ramos, E., Anzaldo-Velázquez, J. E., Aguirre-Ortega, J., \& Martínez Virgen, M. (2014). Bases para el análisis del entorno de la apicultura en Nayarit. Revista Mexicana de Agronegocios, 34, 816-828.

- Ulmer, J., Travadelo, M., Caporgno, J., \& Castignani, H. (2011). Identificación y caracterización de los modelos de producción apícola representativos de la zona Central de la provincia de Santa Fe. Ciencias Agronómicas, 18, 043-049. 